\title{
Almost-distribution cosine functions and integrated cosine functions
}

\author{
by \\ Pedro J. Miana (Zaragoza)
}

\begin{abstract}
We introduce the notion of almost-distribution cosine functions in a setting similar to that of distribution semigroups defined by Lions. We prove general results on equivalence between almost-distribution cosine functions and $\alpha$-times integrated cosine functions.
\end{abstract}

Introduction. Integrated cosine functions of operators in Banach spaces have been introduced to study abstract second order "ill-posed" Cauchy problems $([11])$. $\alpha$-Times integrated cosine functions were introduced for $\alpha \in \mathbb{N}$ in [1] and later defined for $\alpha \geq 0$ ([11], [12]). 0-times integrated cosine functions are usual cosine functions. Differential operators in Euclidean spaces are examples of $\alpha$-times integrated cosine functions (see [1] and [11]).

E. Marschall considered vector-valued cosine transforms defined by cosine functions ([5]) and he applied them to study spectral properties and the spectral mapping theorem for cosine functions. The present author worked with trigonometric convolution products, cosine functions and sine functions (1-times integrated cosine functions) to define vector-valued cosine and sine transforms ([6]). Almost-distribution cosine function is a new related concept, closer to distribution semigroups defined by J.-L. Lions [4].

Every $\alpha$-times integrated cosine function leads to an almost-distribution cosine function of order $\alpha$. We apply Banach algebras $\mathcal{T}_{+}^{(\alpha)}\left(\tau_{\alpha}, *_{c}\right)$ with respect to cosine convolution product, which are defined using Weyl fractional derivation. Conversely, almost-distribution cosine functions of order $\alpha$ define integrated cosine functions. These ideas also hold in the case of integrated semigroups and distribution semigroups (see [7]). The main facts of fractional calculi are presented in the first section.

2000 Mathematics Subject Classification: 47D09, 47D99, 26A33.

Key words and phrases: integrated cosine function, cosine transform, fractional calculus, cosine convolution product.

Partially supported by the Spanish Project BFM2001-1793, MCYT, DGI-FEDER and the DGA project E-12/25. 
Notation. $\Re z$ is the real part of a complex number $z ; \Gamma$ is the Gamma function; $X, Y$ are Banach spaces; $X \hookrightarrow Y$ means a continuous embedding; $T: X \rightarrow Y$ is a bounded linear map from $X$ to $Y$ and $\operatorname{ker} T$ is the kernel of $T ; \mathcal{B}(X)$ is the set of bounded linear operators on $X ; C_{\alpha}$ is a constant which may depend on $\alpha$.

1. Fractional Banach algebras on $\mathbb{R}^{+}$. In this section we review some results and also prove new ones about Weyl fractional calculus (see Theorem 3). Let $\tau_{0}:[0, \infty) \rightarrow[0, \infty)$ be a measurable function on $[0, \infty)$ such that $\tau_{0}(t+s) \leq C_{0} \tau_{0}(t) \tau_{0}(s)$ and $\tau_{0}(t-s) \leq C_{0} \tau_{0}(t) \tau_{0}(s)$ for any $0<s<t$ and $C_{0}>0$. Then $L^{1}\left(\mathbb{R}^{+}, \tau_{0}\right)$ is the Banach space of functions $f$ with $\|f\|_{\tau_{0}}:=\int_{0}^{\infty}|f(t)| \tau_{0}(t) d t<\infty$. Take $f, g \in L^{1}\left(\mathbb{R}^{+}, \tau_{0}\right)$. Then $f * g, f \circ g$ $\in L^{1}\left(\mathbb{R}^{+}, \tau_{0}\right)$, where

$$
f * g(t):=\int_{0}^{t} f(t-s) g(s) d s, \quad f \circ g(t):=\int_{t}^{\infty} f(s-t) g(s) d s, \quad t \geq 0 .
$$

The cosine convolution product $f *_{c} g$ is defined by $f *_{c} g:=\frac{1}{2}(f * g+f \circ g+$ $g \circ f$ ) (see [10]). Let $\mathcal{D}_{+}$be the class of $\mathcal{C}^{\infty}$ functions of compact support on $[0, \infty)$. For $f \in \mathcal{D}_{+}$and $\alpha>0$, the Weyl fractional integral $W_{+}^{-\alpha} f$ of order $\alpha$ is defined by

$$
W_{+}^{-\alpha} f(t):=\frac{1}{\Gamma(\alpha)} \int_{t}^{\infty}(s-t)^{\alpha-1} f(s) d s, \quad t \geq 0,
$$

and the Weyl fractional derivative $W_{+}^{\alpha} f$ of order $\alpha$ is given by

$$
W_{+}^{\alpha} f(t):=\frac{(-1)^{n}}{\Gamma(n-\alpha)} \frac{d^{n}}{d t^{n}} \int_{t}^{\infty}(s-t)^{n-\alpha-1} f(s) d s, \quad t \geq 0,
$$

with $n=[\alpha]+1$. It is known that $W_{+}^{\alpha+\beta}=W_{+}^{\alpha}\left(W_{+}^{\beta}\right)$ for any $\alpha, \beta \in \mathbb{R}$, where $W_{+}^{0}=$ Id is the identity operator $([8])$. The following proposition can be checked directly:

Proposition 1. Given $f, g \in \mathcal{D}_{+}$and $\alpha \in \mathbb{R}$, we have

(i) $W_{+}^{\alpha}(f \circ g)=f \circ W_{+}^{\alpha} g$.

(ii) $W_{+}^{\alpha}\left(f *_{c} g\right)=\frac{1}{2}\left(W_{+}^{\alpha}(f * g)+f \circ W_{+}^{\alpha} g+g \circ W_{+}^{\alpha} f\right)$.

Weyl fractional calculus can also be applied to functions not belonging to $\mathcal{D}_{+}$(see [8, p. 248]). For example, let $f$ and $g$ be measurable functions on $[0, \infty)$ such that $W_{+}^{-\alpha} f$ exists and $g=W_{+}^{-\alpha} f$ a.e. Then we set $W_{+}^{\alpha} g=f$. For example, the Bochner-Riesz functions $\left(R_{t}^{\theta}\right)_{t>0}$ defined by

$$
R_{t}^{\theta}(s)=\frac{(t-s)^{\theta}}{\Gamma(\theta+1)} \chi_{(0, t)}(s) \quad \text { for } t>0 \text { and } \theta>-1
$$

satisfy $W_{+}^{\alpha} R_{t}^{\theta}=R_{t}^{\theta-\alpha}$ for $\theta+1>\alpha \geq 0$. 
We recall that $\Omega_{\alpha}$ is the set of nondecreasing continuous functions $\tau_{\alpha}$ on $(0, \infty)$ such that $\inf _{u>0} u^{-\alpha} \tau_{\alpha}(u)>0$ and there exists a constant $C_{\alpha}>0$ with

$$
\int_{[0, r] \cup[s, s+r]} u^{\alpha-1} \tau_{\alpha}(r+s-u) d u \leq C_{\alpha} \tau_{\alpha}(r) \tau_{\alpha}(s), \quad 0 \leq r \leq s
$$

(see [2]). The functions $\tau_{\alpha}(t)=t^{\alpha} ; t^{\beta}(1+t)^{\nu}$ with $\beta \in[0, \alpha]$ and $\nu \geq \alpha-\beta$; $t^{\beta} e^{\tau t}$ with $\tau>0$ and $\beta \in[0, \alpha]$, all belong to $\Omega_{\alpha}$. If $\tau_{\alpha} \in \Omega_{\alpha}$ then $\tau_{\nu} \in \Omega_{\nu}$, where $\tau_{\nu}(t):=t^{\nu-\alpha} \tau_{\alpha}(t)$ for $t \geq 0$ and $\nu \geq \alpha$. The subset of functions $\tau_{\alpha}(t)=t^{\alpha} w_{0}(t)$, where $w_{0}$ is a continuous nondecreasing weight, is denoted by $\Omega_{\alpha}^{h}$ (see [2] for more details).

Lemma 2. Let $\alpha>0$ and $\tau_{\alpha} \in \Omega_{\alpha}$. If $0<s<t$ then
(i) $\int_{t-s}^{t}(r-t+s)^{\alpha-1} \tau_{\alpha}(r) d r \leq C_{\alpha} \tau_{\alpha}(t) \tau_{\alpha}(s)$.
(ii) $\int_{0}^{s}(r+t-s)^{\alpha-1} \tau_{\alpha}(r) d r \leq C_{\alpha} \tau_{\alpha}(t) \tau_{\alpha}(s)$.

Proof. As $\tau_{\alpha}$ is nondecreasing, we get

$$
\begin{aligned}
\int_{t-s}^{t}(r-t+s)^{\alpha-1} \tau_{\alpha}(r) d r & \leq \tau_{\alpha}(t) \int_{t-s}^{t}(r-t+s)^{\alpha-1} d r \\
& =\frac{\tau_{\alpha}(t)}{\alpha} s^{\alpha} \leq C_{\alpha} \tau_{\alpha}(t) \tau_{\alpha}(s) .
\end{aligned}
$$

(ii) is proven in a similar way.

In [2, Propositions 1.4 and 1.5] the convolution product $*$ is considered, leading to results similar to Theorem 3 below. We denote by $\operatorname{Mul}(\mathcal{A})$ the set of multipliers of a Banach algebra $\mathcal{A}$.

Theorem 3. Let $\alpha>0$ and $\tau_{\alpha} \in \Omega_{\alpha}$. The expression

$$
q_{\tau_{\alpha}}(f):=\frac{1}{\Gamma(\alpha+1)} \int_{0}^{\infty} \tau_{\alpha}(t)\left|W_{+}^{\alpha} f(t)\right| d t, \quad f \in \mathcal{D}_{+},
$$

defines a norm on $\mathcal{D}_{+}$. Moreover, $q_{\tau_{\alpha}}\left(f *_{c} g\right) \leq C_{\alpha} q_{\tau_{\alpha}}(f) q_{\tau_{\alpha}}(g)$ for $f, g$ $\in \mathcal{D}_{+}$, and $C_{\alpha}>0$ is independent of $f$ and $g$. Denote by $\mathcal{T}_{+}^{(\alpha)}\left(\tau_{\alpha}, *_{c}\right)$ the Banach algebra obtained as the completion of $\mathcal{D}_{+}$in the norm $q_{\tau_{\alpha}}$.

(i) $\mathcal{T}_{+}^{(\alpha)}\left(\tau_{\alpha}, *_{c}\right) \hookrightarrow \mathcal{T}_{+}^{(\alpha)}\left(t^{\alpha}, *_{c}\right) \hookrightarrow L^{1}\left(\mathbb{R}^{+}, *_{c}\right)$.

(ii) If $\beta>\alpha>0$, and $\tau_{\beta} \in \Omega_{\beta}$ is such that

$$
\frac{1}{\Gamma(\beta-\alpha) \Gamma(\alpha+1)} \int_{0}^{t}(t-s)^{\beta-\alpha-1} \tau_{\alpha}(s) d s \leq \frac{1}{\Gamma(\beta+1)} \tau_{\beta}(t), \quad t \geq 0,
$$


then $\mathcal{T}_{+}^{(\beta)}\left(\tau_{\beta}, *_{c}\right) \hookrightarrow \mathcal{T}_{+}^{(\alpha)}\left(\tau_{\alpha}, *_{c}\right)$; in particular $\mathcal{T}_{+}^{(\beta)}\left(t^{\beta}, *_{c}\right) \hookrightarrow$ $\mathcal{T}_{+}^{(\alpha)}\left(t^{\alpha}, *_{c}\right)$.

(iii) $R_{t}^{\nu-1} \in \mathcal{T}_{+}^{(\alpha)}\left(\tau_{\alpha}, *_{c}\right)$ for $t>0$ and $\nu>\alpha$, and $q_{\tau_{\alpha}}\left(R_{t}^{\nu-1}\right) \leq C_{\nu, \alpha} t^{\nu-\alpha} \tau_{\alpha}(t)$ for $t>0$, where $C_{\nu, \alpha}>0$ is independent of $t$.

(iv) $R_{t}^{\alpha-1} \in \operatorname{Mul}\left(\mathcal{T}_{+}^{(\alpha)}\left(\tau_{\alpha}, *_{c}\right)\right)$ and $\left\|R_{t}^{\alpha-1}\right\|_{\operatorname{Mul}\left(\mathcal{T}_{+}^{(\alpha)}\left(\tau_{\alpha}, *_{c}\right)\right)} \leq C \tau_{\alpha}(t)$ for $t>0$.

Proof. Clearly $q_{\tau_{\alpha}}$ is a norm on $\mathcal{D}_{+}$and

$$
q_{\tau_{\alpha}}\left(f *_{c} g\right) \leq \frac{1}{2}\left(q_{\tau_{\alpha}}(f * g)+q_{\tau_{\alpha}}(f \circ g)+q_{\tau_{\alpha}}(g \circ f)\right) .
$$

As $q_{\tau_{\alpha}}(f * g) \leq C_{\alpha} q_{\tau_{\alpha}}(f) q_{\tau_{\alpha}}(g)$ (see [2, Proposition 1.4]), it is enough to check $q_{\tau_{\alpha}}(f \circ g) \leq C_{\alpha} q_{\tau_{\alpha}}(f) q_{\tau_{\alpha}}(g)$. We apply Proposition 1(i), the Fubini theorem and Lemma 2 to get

$$
\begin{aligned}
q_{\tau_{\alpha}}(f \circ g) \leq & \int_{0}^{\infty} \tau_{\alpha}(t) \int_{t}^{\infty} \frac{1}{\Gamma(\alpha)} \int_{s-t}^{\infty}(u-s+t)^{\alpha-1}\left|W_{+}^{\alpha} f(u)\right| d u\left|W_{+}^{\alpha} g(s)\right| d s d t \\
\leq & \frac{1}{\Gamma(\alpha)} \int_{0}^{\infty}\left|W_{+}^{\alpha} g(s)\right| \int_{0}^{s}\left|W_{+}^{\alpha} f(u)\right| \int_{s-u}^{s}(u-s+t)^{\alpha-1} \tau_{\alpha}(t) d t d u d s \\
& +\frac{1}{\Gamma(\alpha)} \int_{0}^{\infty}\left|W_{+}^{\alpha} g(s)\right| \int_{s}^{\infty}\left|W_{+}^{\alpha} f(u)\right| \int_{0}^{s}(u-s+t)^{\alpha-1} \tau_{\alpha}(t) d t d u d s \\
\leq & C_{\alpha} q_{\tau_{\alpha}}(f) q_{\tau_{\alpha}}(g) .
\end{aligned}
$$

(i) and (ii) are checked directly and (iii) appears in [2].

(iv) Take $f \in \mathcal{D}_{+}$; we shall prove $R_{t}^{\alpha-1} * f \in \mathcal{T}_{+}^{(\alpha)}\left(\tau_{\alpha}, *_{c}\right)$ for any $t>0$. By [2, Proposition 1.5], $R_{t}^{\alpha-1} \in \operatorname{Mul}\left(\mathcal{T}_{+}^{(\alpha)}\left(\tau_{\alpha}, *\right)\right)$, and it is enough to prove $R_{t}^{\alpha-1} \circ f, f \circ R_{t}^{\alpha-1} \in \mathcal{T}_{+}^{(\alpha)}\left(\tau_{\alpha}, *_{c}\right)$. Since $W^{\alpha}\left(R_{t}^{\alpha-1} \circ f\right)=R_{t}^{\alpha-1} \circ W_{+}^{\alpha} f$ and $W_{+}^{\alpha}\left(f \circ R_{t}^{\alpha-1}\right)(s)=f(s+t)$ for $s, t>0$, we use again Lemma 2 to obtain $R_{t}^{\alpha-1} \in \operatorname{Mul}\left(\mathcal{T}_{+}^{(\alpha)}\left(\tau_{\alpha}, *_{c}\right)\right)$, and $\left\|R_{t}^{\alpha-1}\right\|_{\operatorname{Mul}\left(\mathcal{T}_{+}^{(\alpha)}\left(\tau_{\alpha}, *_{c}\right)\right)} \leq C \tau_{\alpha}(t)$ for $t>0$.

If $\tau_{\alpha} \in \Omega_{\alpha}^{h}$ with $\alpha \geq 0$, the algebra $\mathcal{T}_{+}^{(\alpha)}\left(\tau_{\alpha}, *_{c}\right)$ has bounded approximate identities (take $\phi \in \mathcal{T}_{+}^{(\alpha)}\left(\tau_{\alpha}\right)$ such that $\int_{0}^{\infty} \phi(t) d t=1$ and consider $\left(\phi_{s}=\right.$ $\left.(1 / s) \phi(\cdot / s))_{0<s<1}\right)$. In general, the algebras $\mathcal{T}_{+}^{(\alpha)}\left(\tau_{\alpha}, *_{c}\right)$ do not have any bounded approximate identity.

2. $\alpha$-Times integrated cosine functions. Given $\alpha>0$, a family $\left(C_{\alpha}(t)\right)_{t \geq 0} \subset \mathcal{B}(X)$ of strongly continuous operators is an $\alpha$-times integrated 
cosine function if $C_{\alpha}(0)=0$ and

$$
\begin{aligned}
2 \Gamma(\alpha) C_{\alpha}(t) & C_{\alpha}(s) x=\left(\int_{t}^{t+s}-\int_{0}^{s}\right)(t+s-r)^{\alpha-1} C_{\alpha}(r) x d r \\
& +\int_{t-s}^{t}(r-t+s)^{\alpha-1} C_{\alpha}(r) x d r+\int_{0}^{s}(r+t-s)^{\alpha-1} C_{\alpha}(r) x d r
\end{aligned}
$$

for all $t>s>0$ and $x \in X$. Every $\alpha$-times integrated cosine function $\left(C_{\alpha}(t)\right)_{t \geq 0}$ yields a $\nu$-times integrated cosine function $\left(C_{\nu}(t)\right)_{t \geq 0}$ defined by

$$
C_{\nu}(t) x:=\frac{1}{\Gamma(\nu-\alpha)} \int_{0}^{t}(t-s)^{\nu-\alpha-1} C_{\alpha}(s) x d s, \quad t \geq 0, x \in X .
$$

0-Times integrated cosine functions are usual cosine functions. An $\alpha$-times integrated cosine function $\left(C_{\alpha}(t)\right)_{t>0}$ is called nondegenerate if $C_{\alpha}(t) x=0$ for all $t \geq 0$ implies that $x=0$. We only consider nondegenerate integrated cosine functions. We define its generator $(A, D(A))$, where $D(A)$ is the set of $x \in X$ such that there exists $y \in X$ satisfying

$$
C_{\alpha}(t) x-\frac{t^{\alpha}}{\Gamma(\alpha+1)} x=\int_{0}^{t}(t-r) C_{\alpha}(r) y d r, \quad t>0,
$$

and $A x:=y$. It is straightforward to check that $(A, D(A))$ is a closed operator. For every $x \in X, \int_{0}^{t}(t-s) C_{\alpha}(s) x d s \in D(A)$ and

$$
C_{\alpha}(t) x=A \int_{0}^{t}(t-s) C_{\alpha}(s) x d s+\frac{t^{\alpha}}{\Gamma(\alpha+1)} x .
$$

If $x \in D(A)$ then $C_{\alpha}(\cdot) x$ is differentiable for $t \geq 0$ and

$$
\begin{aligned}
\frac{d}{d t} C_{\alpha}(t) x & =\int_{0}^{t} C_{\alpha}(s) A x d s+\frac{t^{\alpha-1}}{\Gamma(\alpha)} x \quad \text { for } \alpha>0 \\
\frac{d}{d t} C_{0}(t) x & =\int_{0}^{t} C_{0}(s) A x d s
\end{aligned}
$$

([9], [11]). If $\left\|C_{\alpha}(t)\right\| \leq C e^{\lambda_{0} t}$ with $C, \lambda_{0} \geq 0$, condition (1) is equivalent (via Laplace transform) to

$$
R\left(\lambda^{2}, A\right) x:=\lambda^{\alpha-1} \int_{0}^{\infty} e^{-\lambda t} C_{\alpha}(t) x d t, \quad x \in X, \Re \lambda^{2}>\lambda_{0},
$$

being a pseudo-resolvent operator, i.e.,

$$
R\left(\lambda^{2}, A\right)-R\left(\mu^{2}, A\right)=\left(\mu^{2}-\lambda^{2}\right) R\left(\lambda^{2}, A\right) R\left(\mu^{2}, A\right), \quad \Re \lambda^{2}, \Re \mu^{2}>\lambda_{0}
$$


(for $\alpha=n$ see [9, Theorem 1.3]). In the nondegenerate case, $\Re \lambda^{2}$ belongs to the resolvent set $\varrho(A)$ and $R\left(\lambda^{2}, A\right)=\left(\lambda^{2}-A\right)^{-1}$.

For $\nu>\alpha,\left(R_{t}^{\nu-1}\right)_{t>0}$ is a $\nu$-times integrated cosine function in $\mathcal{T}_{+}^{(\alpha)}\left(\tau_{\alpha}, *_{c}\right)$. This may be proved using Laplace transform. This family is the canonical integrated cosine function (see Theorem 4(i)). In the case of cosine functions, this result appears in [5].

TheOREM 4. Let $\left(C_{\alpha}(t)\right)_{t \geq 0}$ be an $\alpha$-times integrated cosine function on $X$ generated by $(A, D(A))$ such that $\left\|C_{\alpha}(t)\right\| \leq C \tau_{\alpha}(t), t \geq 0$, where $\tau_{\alpha} \in \Omega_{\alpha}$. Then the $\operatorname{map} \mathcal{C}_{+}: \mathcal{T}_{+}^{(\alpha)}\left(\tau_{\alpha}, *_{c}\right) \rightarrow \mathcal{B}(X)$ given by

$$
\mathcal{C}_{+}(f) x=\int_{0}^{\infty} W_{+}^{\alpha} f(t) C_{\alpha}(t) x d t, \quad f \in \mathcal{T}_{+}^{(\alpha)}\left(\tau_{\alpha}, *_{c}\right), x \in X,
$$

is a continuous Banach algebra homomorphism. Moreover,

(i) If $\nu>\alpha$ and $\left(R_{t}^{\nu-1}\right)_{t>0}$ are the Bochner-Riesz functions then $C_{\nu}(t)$ $=\mathcal{C}_{+}\left(R_{t}^{\nu-1}\right)$, where $\left(C_{\nu}(t)\right)_{t \geq 0}$ is defined as in (2) and

$$
\int_{0}^{\infty} W_{+}^{\alpha} f(t) C_{\alpha}(t) x d t=\int_{0}^{\infty} W_{+}^{\nu} f(t) C_{\nu}(t) x d t, \quad x \in X,
$$

for $f \in \mathcal{T}_{+}^{(\nu)}\left(\tau_{\nu}, *_{c}\right) \hookrightarrow \mathcal{T}_{+}^{(\alpha)}\left(\tau_{\alpha}, *_{c}\right)$ and $\tau_{\nu}(t):=t^{\nu-\alpha} \tau_{\alpha}(t)$ for $t \geq 0$.

(ii) If $x \in D(A)$ then $C_{\alpha}(\cdot) x$ is differentiable for any $t \geq 0$, and for $f \in \mathcal{D}_{+}$,

$$
\begin{aligned}
\int_{0}^{\infty} W_{+}^{\alpha} f(t) \frac{d}{d t} C_{\alpha}(t) x d t & =A \mathcal{C}_{+}\left(W_{+}^{-1} f\right) x+f(0) x \quad \text { for } \alpha>0 \\
\int_{0}^{\infty} f(t) \frac{d}{d t} C_{0}(t) x d t & =A \mathcal{C}_{+}\left(W_{+}^{-1} f\right) x .
\end{aligned}
$$

Proof. We suppose $\alpha>0$. As $\left\|C_{\alpha}(t)\right\| \leq C \tau_{\alpha}(t)$ for any $t \geq 0$, the expression

$$
\mathcal{C}_{+}(f) x:=\int_{0}^{\infty} W_{+}^{\alpha} f(t) C_{\alpha}(t) x d t, \quad f \in \mathcal{T}_{+}^{(\alpha)}\left(\tau_{\alpha}, *_{c}\right), x \in X,
$$

defines a continuous linear homomorphism $\mathcal{C}_{+}: \mathcal{T}_{+}^{(\alpha)}\left(\tau_{\alpha}, *_{c}\right) \rightarrow \mathcal{B}(X)$. Indeed, for $f, g \in \mathcal{D}_{+}$we shall prove $\mathcal{C}_{+}\left(f *_{c} g\right)=\mathcal{C}_{+}(f) \mathcal{C}_{+}(g)$. By Proposition 1(ii), we have

$$
\begin{aligned}
\Gamma(\alpha) \mathcal{C}_{+}\left(f *_{c} g\right) x & =\Gamma(\alpha) \int_{0}^{\infty} W_{+}^{\alpha}\left(f *_{c} g\right)(t) C_{\alpha}(t) x d t \\
& =\frac{\Gamma(\alpha)}{2} \int_{0}^{\infty}\left(W_{+}^{\alpha}(f * g)+f \circ W_{+}^{\alpha} g+g \circ W_{+}^{\alpha} f\right)(t) C_{\alpha}(t) x d t
\end{aligned}
$$


for $x \in X$. Using [7, Proposition 1.1] and Fubini theorem, we get

$$
\begin{aligned}
& \Gamma(\alpha) \int_{0}^{\infty} W_{+}^{\alpha}(f * g)(t) C_{\alpha}(t) x d t \\
& =\int_{0}^{\infty} W_{+}^{\alpha} g(r) \int_{0}^{r} W_{+}^{\alpha} f(s)\left(\left(\int_{r}^{s+r}-\int_{0}^{s}\right)(s+r-t)^{\alpha-1} C_{\alpha}(t) x d t\right) d s d r \\
& \quad+\int_{0}^{\infty} W_{+}^{\alpha} g(r) \int_{r}^{\infty} W_{+}^{\alpha} f(s)\left(\left(\int_{s}^{s+r}-\int_{0}^{r}\right)(s+r-t)^{\alpha-1} C_{\alpha}(t) x d t\right) d s d r .
\end{aligned}
$$

Again by the Fubini theorem,

$$
\int_{0}^{\infty}\left(f \circ W_{+}^{\alpha} g\right)(t) C_{\alpha}(t) x d t=\int_{0}^{\infty} W_{+}^{\alpha} g(r) \int_{0}^{r} f(r-t) C_{\alpha}(t) x d t d r
$$

and using $f(r-t)=W_{+}^{-\alpha}\left(W_{+}^{\alpha} f\right)(r-t)$, we obtain

$$
\begin{aligned}
\Gamma(\alpha) \int_{0}^{\infty}\left(f \circ W_{+}^{\alpha} g\right)(t) C_{\alpha}(t) x d t & \\
= & \int_{0}^{\infty} W_{+}^{\alpha} g(r) \int_{0}^{r} W_{+}^{\alpha} f(s) \int_{r-s}^{r}(s-r+t)^{\alpha-1} C_{\alpha}(t) x d t d s d r \\
& +\int_{0}^{\infty} W_{+}^{\alpha} g(r) \int_{r}^{\infty} W_{+}^{\alpha} f(s) \int_{0}^{r}(t+s-r)^{\alpha-1} C_{\alpha}(t) x d t d s d r .
\end{aligned}
$$

In the same way, we also get

$$
\begin{aligned}
\Gamma(\alpha) \int_{0}^{\infty}\left(g \circ W_{+}^{\alpha} f\right)(t) C_{\alpha}(t) x d t \\
=\int_{0}^{\infty} W_{+}^{\alpha} g(r) \int_{r}^{\infty} W_{+}^{\alpha} f(s) \int_{s-r}^{r}(s-r+t)^{\alpha-1} C_{\alpha}(t) x d t d s d r \\
\quad+\int_{0}^{\infty} W_{+}^{\alpha} g(r) \int_{0}^{r} W_{+}^{\alpha} f(s) \int_{0}^{r}(t+s-r)^{\alpha-1} C_{\alpha}(t) x d t d s d r .
\end{aligned}
$$

We join these six summands to conclude that

$$
\begin{aligned}
\Gamma(\alpha) \mathcal{C}_{+}\left(f *_{c} g\right) x= & \Gamma(\alpha) \int_{0}^{\infty} W_{+}^{\alpha} g(r) C_{\alpha}(r) \int_{0}^{r} W_{+}^{\alpha} f(s) C_{\alpha}(s) x d s d r \\
& +\Gamma(\alpha) \int_{0}^{\infty} W_{+}^{\alpha} g(r) C_{\alpha}(r) \int_{r}^{\infty} W_{+}^{\alpha} f(s) C_{\alpha}(s) x d s d r \\
= & \Gamma(\alpha) \mathcal{C}_{+}(g) \mathcal{C}_{+}(f) x .
\end{aligned}
$$

Parts (i) and (ii) are checked using (3) and (4). 


\section{Almost-distribution cosine functions}

Definition 5. An almost-distribution cosine function on $X$ is a continuous linear map $\mathcal{C}_{+}: \mathcal{D}_{+} \rightarrow \mathcal{B}(X)$ with the following properties:

(i) $\mathcal{C}_{+}\left(f *_{c} g\right)=\mathcal{C}_{+}(f) \mathcal{C}_{+}(g)$ for any $f, g \in \mathcal{D}_{+}$.

(ii) $\bigcap\left\{\operatorname{ker} \mathcal{C}_{+}(f) \mid f \in \mathcal{D}_{+}\right\}=\{0\}$.

The generator $(A, D(A))$ of an almost-distribution cosine function $\mathcal{C}_{+}$is defined by: $x \in D(A)$ when there exists $y \in X$ such that $\mathcal{C}_{+}(f) y=\mathcal{C}_{+}\left(f^{\prime \prime}\right) x+$ $f^{\prime}(0) x$ for any $f \in \mathcal{D}_{+}$, and $A x:=y$. The generator $(A, D(A))$ is well defined, closed, $\mathcal{C}_{+}\left(\mathcal{D}_{+}\right) X \subset D(A)$, and $A \mathcal{C}_{+}(f) x=\mathcal{C}_{+}\left(f^{\prime \prime}\right) x+f^{\prime}(0) x$ for any $f \in \mathcal{D}_{+}$and $x \in X$. If $x \in D(A)$ and $f \in \mathcal{D}_{+}$then $A \mathcal{C}_{+}(f) x=\mathcal{C}_{+}(f) A x$. An almost-distribution cosine function $\mathcal{C}_{+}$is said to be of order $\alpha>0$ and growth $\tau_{\alpha} \in \Omega_{\alpha}$ if $\mathcal{C}_{+}$, regarded as defined on $\mathcal{D}_{+}$, can be extended to a continuous linear map $\mathcal{C}_{+}: \mathcal{T}_{+}^{(\alpha)}\left(\tau_{\alpha}, *_{c}\right) \rightarrow \mathcal{B}(X)$. We connect this kind of almost-distribution cosine functions and integrated cosine functions.

TheOREM 6. Let $\left(C_{\alpha}(t)\right)_{t \geq 0}$ be an $\alpha$-times integrated cosine function on $X$ generated by $(A, D(A))$ such that $\left\|C_{\alpha}(t)\right\| \leq C \tau_{\alpha}(t),\left(t \geq 0, \tau_{\alpha} \in \Omega_{\alpha}\right)$. Then $(A, D(A))$ generates an almost-distribution cosine function, $\mathcal{C}_{+}: \mathcal{T}_{+}^{(\alpha)}\left(\tau_{\alpha}, *_{c}\right)$ $\rightarrow \mathcal{B}(X)$

$$
\mathcal{C}_{+}(f) x=\int_{0}^{\infty} W_{+}^{\alpha} f(t) C_{\alpha}(t) x d t, \quad f \in \mathcal{T}_{+}^{(\alpha)}\left(\tau_{\alpha}, *_{c}\right), x \in X,
$$

of order $\alpha>0$ and growth $\tau_{\alpha} \in \Omega_{\alpha}$.

Proof. Condition (i) of Definition 5 is proven in Theorem 4. Take $x \in$ $\bigcap_{f \in \mathcal{D}_{+}} \operatorname{ker} \mathcal{C}_{+}(f)$. Then $C_{\alpha+1}(t) x=0$ and $C_{\alpha}(t) x=\frac{d}{d t} C_{\alpha+1}(t) x=0$ for any $t>0$, so we conclude that $x=0$ and $\mathcal{C}_{+}$is an almost-distribution cosine function.

Let $(B, D(B))$ be the generator of $\mathcal{C}_{+}$and $x \in D(A)$ with $y=A x$. Applying Theorem 4(i) and (3), we see that, for $f \in \mathcal{D}_{+}$,

$$
\begin{aligned}
\mathcal{C}_{+}(f) y & =\int_{0}^{\infty} W_{+}^{\alpha+2} f(t) C_{\alpha+2}(t) y d t=\int_{0}^{\infty} W_{+}^{\alpha+1} f(t) \int_{0}^{t}(t-s) C_{\alpha}(s) y d s d t \\
& =\int_{0}^{\infty} W_{+}^{\alpha+2} f(t)\left(C_{\alpha}(t) x-\frac{t^{\alpha} x}{\Gamma(\alpha+1)}\right) d t \\
& =\int_{0}^{\infty} W_{+}^{\alpha} f^{\prime \prime}(t) C_{\alpha}(t) x d t-\int_{0}^{\infty} W_{+}^{\alpha+2} f(t) \frac{t^{\alpha} x}{\Gamma(\alpha+1)} d t \\
& =\mathcal{C}_{+}\left(f^{\prime \prime}\right) x+f^{\prime}(0) x .
\end{aligned}
$$

Hence $x \in D(B)$ and $B x=A x$. Now we take $x \in D(B)$ and $y=B x$ and 
since $\left(R_{t}^{\alpha+2}\right)_{t>0} \subset \mathcal{T}_{+}^{(\alpha)}\left(\tau_{\alpha}\right)$, we get

$$
C_{\alpha+3}(t) y=C_{\alpha+1}(t) x-\frac{t^{\alpha+1}}{\Gamma(\alpha+2)} x .
$$

Differentiating gives $C_{\alpha+2}(t) y=C_{\alpha}(t) x-\frac{t^{\alpha}}{\Gamma(\alpha+1)} x$. Thus $x \in D(A)$ and $A x=B x$.

Now we prove the first converse to Theorem 6 .

TheOREM 7. Given $\alpha \geq 0, \tau_{\alpha} \in \Omega_{\alpha}$, and an almost-distribution cosine function $\mathcal{C}_{+}: \mathcal{T}_{+}^{(\alpha)}\left(\tau_{\alpha}, *_{c}\right) \rightarrow \mathcal{B}(X)$ generated by $(A, D(A))$. Then for any $\nu>\alpha,(A, D(A))$ generates a $\nu$-times integrated cosine function $\left(C_{\nu}(t)\right)_{t \geq 0}$ such that $\left\|C_{\nu}(t)\right\| \leq C_{\nu} \tau_{\nu}(t)$ with $\tau_{\nu}(t)=t^{\nu-\alpha} \tau_{\alpha}(t)$ for any $t \geq 0$, and

$$
\mathcal{C}_{+}(f) x=\int_{0}^{\infty} W_{+}^{\nu} f(t) C_{\nu}(t) x d t, \quad f \in \mathcal{T}_{+}^{(\nu)}\left(\tau_{\nu}, *_{c}\right), x \in X .
$$

Proof. Take $\nu>\alpha$. By Theorem 3(iii), the Bochner-Riesz functions $\left(R_{t}^{\nu-1}\right)_{t \geq 0}$ belong to $\mathcal{T}_{+}^{(\alpha)}\left(\tau_{\alpha}, *_{c}\right)$ with $\nu>\alpha$ and $q_{\tau_{\alpha}}\left(R_{t}^{\nu-1}\right) \leq C_{\nu, \alpha} t^{\nu-\alpha} \tau_{\alpha}(t)$ for $t \geq 0$. Moreover, $\left(R_{t}^{\nu-1}\right)_{t \geq 0}$ is a $\nu$-times integrated cosine function in $\mathcal{T}_{+}^{(\alpha)}\left(\tau_{\alpha}, *_{c}\right)$. We define $C_{\nu}(t):=\mathcal{C}_{+}\left(R_{t}^{\nu-1}\right)$ for any $t \geq 0$. It is clear that $\left(C_{\nu}(t)\right)_{t>0}$ is a $\nu$-times integrated cosine function and by the continuity of $\mathcal{C}_{+},\left\|C_{\nu}(t)\right\| \leq C_{\nu, \alpha} t^{\nu-\alpha} \tau_{\alpha}(t)$ for any $t \geq 0$. Take now $\tau_{\nu}(t):=t^{\nu-\alpha} \tau_{\alpha}(t)$. Then $\tau_{\nu} \in \Omega_{\nu}$ and by Theorem 6 , there exists $\mathcal{C}_{+}^{\prime}: \mathcal{T}_{+}^{(\nu)}\left(\tau_{\nu}, *_{c}\right) \rightarrow \mathcal{B}(X)$ such that

$$
\mathcal{C}_{+}^{\prime}(f) x=\int_{0}^{\infty} W_{+}^{\nu} f(t) C_{\nu}(t) x d t, \quad f \in \mathcal{T}_{+}^{(\nu)}\left(\tau_{\nu}, *_{c}\right), x \in X .
$$

As $\mathcal{T}_{+}^{(\nu)}\left(\tau_{\nu}, *_{c}\right) \hookrightarrow \mathcal{T}_{+}^{(\alpha)}\left(\tau_{\alpha}, *_{c}\right)$, and $\mathcal{C}_{+}$is continuous, we have

$$
\mathcal{C}_{+}^{\prime}(f) x=\int_{0}^{\infty} W_{+}^{\nu} f(t) \mathcal{C}_{+}\left(R_{t}^{\nu-1}\right) x d t=\mathcal{C}_{+}\left(\int_{0}^{\infty} W_{+}^{\nu} f(t) R_{t}^{\nu-1} d t\right) x=\mathcal{C}_{+}(f) x
$$

for any $f \in \mathcal{T}_{+}^{(\nu)}\left(\tau_{\nu}, *_{c}\right)$ and $x \in X$. If $(B, D(B))$ generates $\left(C_{\nu}(t)\right)_{t>0}$ then it generates $\mathcal{C}_{+}^{\prime}$ (Theorem 6), and $(A, D(A))=(B, D(B))$.

By Theorem 3(iv), the Bochner-Riesz functions $\left(R_{t}^{\alpha-1}\right)_{t>0}$ are multipliers of the algebra $\mathcal{T}^{(\alpha)}\left(\tau_{\alpha}, *_{c}\right)$ for any $t>0$ and $\left(R_{t}^{\alpha-1}\right)_{t>0}$ is an $\alpha$-times integrated cosine function in $\operatorname{Mul}\left(\mathcal{T}^{(\alpha)}\left(\tau_{\alpha}, *_{c}\right)\right)$. If $\mathcal{T}_{+}^{(\alpha)}\left(\tau_{\alpha}, *_{c}\right)$ has a bounded approximate identity, then Cohen's factorization theorem holds, and we may define $\left(\mathcal{C}_{+}\left(R_{t}^{\alpha-1}\right)\right)_{t \geq 0}$. We get the second converse to Theorem 6 . The proof is similar to [7, Theorem 4.9].

Theorem 8. Let $\alpha \geq 0, \tau_{\alpha} \in \Omega_{\alpha}^{h}$ and $(A, D(A))$ a closed and densely defined operator on $X$. The following conditions are equivalent. 
(i) $(A, D(A))$ generates an $\alpha$-times integrated cosine function $\left(C_{\alpha}(t)\right)_{t \geq 0}$ such that $\left\|C_{\alpha}(t)\right\| \leq C_{\alpha} \tau_{\alpha}(t)$ for any $t \geq 0$.

(ii) $(A, D(A))$ generates an almost-distribution cosine function $\mathcal{C}_{+}$of order $\alpha>0$ and growth $\tau_{\alpha}$ such that $\mathcal{C}_{+}\left(\mathcal{D}_{+}\right)(X)$ is dense in $X$.

\section{References}

[1] W. Arendt and H. Kellerman, Integrated solutions of Volterra integrodifferential equations and applications, in: Integrodifferential Equations (Trento, 1987), G. Da Prato and M. Iannelli (eds.), Pitman Res. Notes Math. Ser. 190, Longman Sci. Tech., Harlow, 1987, 21-51.

[2] J. E. Galé and P. J. Miana, One parameter groups of regular quasimultiplier, preprint.

[3] Y.-C. Li and S.-Y. Shaw, On generators of integrated $C$-semigroups and $C$-cosine functions, Semigroup Forum 47 (1993), 29-35.

[4] J.-L. Lions, Semi-groupes distributions, Portugal. Math. 19 (1960), 141-164.

[5] E. Marschall, On the functional-calculus of non-quasianalytic groups of operators and cosine functions, Rend. Circ. Mat. Palermo 35 (1986), 58-81.

[6] P. J. Miana, Vectorial cosine and sine transforms, preprint.

[7] - $\alpha$-Times integrated semigroups and fractional derivation, Forum Math. 14 (2002), $23-46$.

[8] K. S. Miller and B. Ross, An Introduction to the Fractional Calculus and Fractional Differential Equations, Wiley, New York, 1993.

[9] S.-Y. Shaw and Y.-C. Li, On $N$-times integrated $C$-cosine functions, in: Evolution Equations, G. Ferreyra et al. (eds.), Lecture Notes in Pure and Appl. Math. 168, Dekker, New York, 1995, 393-406.

[10] I. N. Sneddon, The Use of Integral Transforms, McGraw-Hill, New York, 1972.

[11] T. J. Xiao and J. Liang, The Cauchy Problem for Higher-Order Abstract Differential Equations, Lecture Notes in Math. 1701, Springer, Berlin, 1998.

[12] G. Yang, $\alpha$-Times integrated cosine function, in: Recent Advances in Differential Equations, Pitman Res. Notes in Math. Ser. 386, Addison-Wesley and Longman, 1998, 199-212.

Departamento de Matemáticas

Universidad de Zaragoza

Zaragoza 50009, Spain

E-mail: pjmiana@unizar.es 